\title{
Valuing Multi-factor Real Options Using an Implied Binomial Tree
}

\author{
Tianyang Wang and James S. Dyer \\ McCombs School of Business, The University of Texas at Austin, Austin, Texas 78712 \\ \{tianyang.wang@phd.mccombs.utexas.edu,jim.dyer@mccombs.utexas.edu\}
}

\begin{abstract}
:
This paper proposes an approach for solving a multi-factor real options problem by approximating the underlying stochastic process with an implied binomial tree. The implied binomial tree is constructed to be consistent with simulated market information. By simulating European option prices as artificial market information, we apply the finance-based Implied Binomial Tree method for real options valuation when the options are contingent on the value of market uncertainties that are not traded assets. Compared to the discrete approximations suggested in the current literature, this method offers a more flexible distribution assumption for project values and provides a simple, computationally efficient and accurate way to price high dimensional real options. For risk managers, it serves as a capital budgeting method for projects with managerial flexibility.
\end{abstract}

Key words: real options; implied binomial tree; multi-factor; simulation

\section{Introduction}

Real options valuation approaches extend financial option pricing theory to situations where flexibility in the deployment of real assets increases the value of a project. Early applications of these ideas to real options directly applied existing financial options valuation methods to projects: stochastic differential equations were used to value simple real options or single-factor lattices were used to approximate the underlying uncertainty of project values. However, these approaches were not appropriate for complex real options valuation because they are difficult to 
apply in practice for large, realistic problems with multiple sources of uncertainty (Borison, 2005).

Decision trees and Monte Carlo simulation have also been proposed as tools for real options valuation. Decision trees can handle multiple sources of uncertainty but are subject to the "curse of dimensionality", and may be particularly difficult to implement when correlation is present in the project uncertainties (Smith and Nau, 1995 and Smith and McCardle, 1999). Monte Carlo simulation is less susceptible to scalability issues for European options, but it is more difficult to implement for complex options with large numbers of alternatives and early exercise dates. Longstaff and Schwartz (2001) provided a hybrid approach of simulation and regression to overcome the early exercise issue. However, the complexity of this approach grows with the number of decisions and alternatives. The incremental modeling and computational requirements of this approach can be significant for compound real options (Smith, 2005).

Some recent developments in real options valuation have focused on approaches to simplify the representation of multiple sources of uncertainty while capturing their impact on the project's cash flows. Copeland and Antikarov (2001) (C\&A) proposed a lattice-based method for multifactor real options valuation. This approach uses Monte Carlo simulation to aggregate a multifactor stochastic process into a consolidated single factor stochastic process based on the project cash flows, and to estimate its parameters. This information is used to generate a binomial lattice that is consistent with some assumed distribution. Smith (2005), Brandão et al. (2005b) (BDH), and Godinho (2006) suggested how to improve the accuracy of the volatility estimation of the C\&A approach and Brandão et al. (2005a, 2005b) suggested the use of binomial decision trees to reproduce these lattices and to allow greater flexibility in modeling and valuing real options. 
If the assumption about the distribution of the stochastic process used to model the project value is valid, the C\&A approach is computationally appealing and accurate; at the same time, the lattice it produces has a simple, intuitive interface which lends itself to practical applications. However, the $\mathrm{C} \& \mathrm{~A}$ and $\mathrm{BDH}$ approaches share a common weakness: as parametric methods, they need to assume a specific distribution for the underlying project value for the whole process or for each time period in order to calibrate the parameter(s) for building the decision lattice/tree.

This paper provides a nonparametric extension of the C\&A approach by allowing project value to follow a generalized diffusion process in which the volatility may vary with time and with the asset prices (Jackwerth, 1999), and therefore offers more modeling flexibility. This is accomplished by using the Implied Binomial Tree (IBT) approach first described by Rubenstein (1994) and widely used to value complex financial options. The main idea of the IBT option pricing model is to introduce option prices to calibrate the tree structure so that it is consistent with these observed market prices. The difficulty of applying the IBT in most real options problems is that there are no tradable options in the market. We solve this problem by simulating option prices to construct the implied binomial tree. By introducing simulated market information into the framework of the IBT, we give this method more flexibility and make it a practical approach for real options valuation. The main advantage of the IBT approach is that it relaxes the requirement of the $\mathrm{C} \& \mathrm{~A}$ and $\mathrm{BDH}$ approaches for specific distribution assumptions for the underlying project value. Additionally, it produces a computationally efficient recombining tree and therefore provides a simple visual interface for real options analysis.

The remainder of this paper is organized as follows: Section 2 discusses the C\&A and BDH approaches and the limitations of their commonly used underlying distribution assumptions. It 
also reviews the IBT method for approximating the underlying stochastic process of a financial option. Section 3 adapts the IBT method to value real options by introducing a few novel features. Section 4 shows a case example and discusses the accuracy of the IBT approximation by comparing the real options value calculated from using the IBT with three alternative methods: the C\&A approach using a Geometric Brownian Motion (GBM) assumption with a constant volatility; the $\mathrm{BDH}$ approach allowing time varying volatilities with a GBM assumption for each time period; and the Longstaff and Schwartz Monte Carlo simulation approach. Section 5 presents a summary of the work and a discussion of its strengths and weaknesses compared to these alternative methods.

\section{Discussion of $\mathrm{C} \& A, B D H$, and IBT approaches}

The C\&A approach combines the use of Monte Carlo simulation and a binomial lattice with risk neutral valuation techniques. It handles multiple factors of uncertainty simultaneously in the simulation of project cash flows to estimate the parameters for a stochastic process that produces a model for the project value, and then applies a binomial lattice approximation to the assumed stochastic process. An example would be the use of the Cox-Ross-Rubenstein (CRR) binomial lattice under the GBM assumption to model the project value, which requires only the estimate of the volatility of the project value.

There are four steps in evaluating the multi-factor real options in the C\&A approach:

\section{$\underline{\text { Step 1. Calculate the Net Present Value }}$}

Calculate the expected net present value of the project without options using a deterministic discounted cash flow analysis. This may be done based on a risk-adjusted discount rate as suggested by $\mathrm{C} \& \mathrm{~A}$ or using a risk neutral approach based on the risk free discount rate as suggested by Smith (2005). 


\section{Step 2. Estimate Parameters}

Use Monte Carlo simulation to estimate the parameters of the stochastic process associated with the project value that depends on the simulated cash flows.

\section{Step 3. Build the Binomial Lattice}

Build a binomial lattice that approximates this stochastic process.

\section{Step 4. Incorporate the Real Options}

Incorporate the related real options in the constructed binomial lattice and value the real options.

Step 2 is a general notion. The parameters to be determined depend on the assumption of which stochastic process provides the best approximation to the change in the project value over time. The lattice constructed in Step 3 reflects the assumed stochastic process.

In the C\&A approach, a specific distribution assumption associated with some stochastic process is needed in estimating the parameters and building the binomial lattice. C\&A and BDH in their related work demonstrate their approaches by assuming that the underlying distribution of the project value in each time period can be approximated with a GBM process with a constant volatility. They estimate the volatility of the value of the project without options using the discounted cash flow model and a Monte Carlo simulation to generate the uncertainties in the project cash flows. Given the present value of these cash flows at time 0 and the estimate of the volatility of this value over time, they construct a CRR recombining lattice or tree. Embedded real options are then calculated based on the lattice or tree. In the following discussion, we do not distinguish between the use of a binomial lattice or tree, since it should be understood that they are alternate problem representations which may be selected based on computational considerations and modeling tastes (Smith (2005) and BDH (2005b)). 
While widely used in the valuation of financial options, the GBM assumption may not be a good approximation to the change in project value in real options problems. First, the GBM assumption implies constant volatility; however, the project cash flows may have time varying volatilities instead. BDH recognized this limitation and discussed estimating the time varying volatilities with the GBM assumption for each time period. Second, the GBM assumption implies that the project value will have a lognormal distribution in each time period. In practice, the project values may have different distributions. C\&A and BDH discussed the use of Arithmetic Brownian Motion or a mean reverting process (Hahn and Dyer, 2008) in modeling the underlying stochastic process with a binomial tree when the GBM assumption is not an appropriate approximation for the project value.

The requirement for specifying a distribution assumption for the underlying project value has some limitations in practice. The project value may not be closely approximated by any simple, well studied stochastic process such as a GBM, Arithmetic Brownian Motion or mean reverting process. In these cases, the constructed binomial tree/lattice structure may be a poor approximation to the stochastic process associated with the project value and consequently the calculated values of the real options will be inaccurate (Smith, 2005). Therefore, a more general approach is desirable.

We propose adapting the C\&A approach by using the more flexible Implied Binomial Tree to solve multi-factor real options problems, which allows us to construct the binomial lattice or tree non-parametrically. With simulated artificial European option prices for the underlying project value used as market information, we adapt the IBT approach to calibrate the tree structure. 
When used to value financial options, the IBT approach creates a flexible binomial tree structure from the market information of the underlying financial asset and its associated financial options. If the tree structure captures the underlying stochastic process accurately, the option prices calculated from the binomial tree method should match these observable market prices. The calibrated binomial tree can then be used to value other derivatives as well.

"From the standpoint of the standard binomial option pricing model, which implies a limiting risk neutral lognormal distribution for the underlying asset, the IBT approach provides the natural (and probably the simplest) way to generalize to arbitrary ending risk neutral probability distributions. ” (Rubinstein, 1994)

Three well known implied binomial tree models are the original development (R-IBT) by Rubinstein (1994), the generalized binomial tree (G-IBT) model proposed by Jackwerth (1997) and the implied volatility tree models proposed by Dupire (1994) and Derman and Kani (1994). These IBT methods are similar in the sense that they all use the prices of an underlying asset and some of its associated options, and they use the risk free rate to infer the unique implied binomial tree. The implied volatility tree models require adjustments to avoid arbitrage violations and are sensitive to the interpolation and extrapolation method. As a contrast, the R-IBT and G-IBT are more stable and easy to implement.

There are two limitations of the R-IBT approach. First, for computational convenience, all risk neutral probabilities of paths leading to the same ending node are assumed to be identical. Second, the IBT is only inferred from options with the same expiration date. As an extension of the R-IBT, the G-IBT drops the assumption that all paths leading to a given node are equally likely by introducing a weight function that governs the backward construction of the implied binomial tree. Additionally, the G-IBT is calibrated using European or American options of 
different maturities. Therefore, it provides more flexibility and accuracy for the calibrated tree structure.

For simplicity, we use the G-IBT to focus the discussion in the next section because of its ease of estimation, flexibility, and stability. Other IBT methods can be applied in a similar way. We refer the interested readers to Jackwerth (1999) for a more detailed discussion of these methods.

\section{Applying IBT in Real Options Valuation}

Two obstacles must be overcome to implement the IBT methods in real options valuation. First, unlike the financial market, there are usually no market prices for the underlying assets; i.e., the investment projects in real options problems. Second, there are no tradable options associated with the underlying investment projects in real options problems. In other words, the market is incomplete.

As suggested and discussed by C\&A (2001), BDH (2005 a) and Smith (2005), the first obstacle can be overcome by the Market Asset Disclaimer (MAD) assumption which serves the role of completing the market. The MAD assumes that the value of the project without options is a traded asset that can be used to form replicating portfolios. Under the MAD assumption, the equilibrium approach to risk-neutral valuation can be applied to investments that cannot be perfectly replicated by trading securities.

The second obstacle is also market related, since the IBT approach requires additional market information to calibrate the tree structure that we typically cannot observe for real options problems. Arnold et al. (2007) showed how to use the IBT for the valuation of real options where the underlying asset is a physical commodity (e.g. gold, oil, and wheat) for which futures options exist but spot options do not. Arnold's approach is constructive but limited for real options 
valuation because it assumes the values of the underlying asset and the commodity are perfectly correlated: e.g., a gold mine and the extracted gold. It is not designed for multi-factor real options valuation and, for many real option projects, the futures option does not exist.

We propose using simulated option prices to calibrate the IBT for real options valuation. We artificially create a sequence of European-style real options with different strike prices and maturities and apply Monte Carlo simulation to calculate the corresponding European-style real options prices. These simulation-based estimates of these simple European-style option values are analogous to the market prices for financial options associated with traded assets. Similar Monte Carlo simulation methods can provide accurate estimates of true market values for financial options, so the use of the simulated European-style real options values is a reasonable way to estimate these values. Then we use the information from these simulated option prices to calibrate the implied binomial tree structure, and use the tree structure to value more complex American-style real options which may be otherwise computationally inefficient for valuation using simulation.

Specifically, we consider a $T$ period project that requires an initial investment $I$ and generates cash flows $X_{t}$ at time $t, t=0, \ldots, T$. $X_{t}$ will vary over time as a stochastic variable reflecting the multiple uncertainties in the project value. For simplicity we adopt the fully risk neutral valuation approach (Smith, 2005) and assume that the cash flows are paid instantaneously at the end of each time period with payout rate $\delta_{t}$ in a manner analogous to stock dividends (C\&A, 2001; BDH, 2005a). The cash flow payout rate $\delta_{t}$ is the fraction of the project value in each period that is paid out as cash in that period, and is determined using the ratio of the expected cash payment to the present value of the sum of the expected values of the remaining cash payments in each period. When uncertainty is reflected in the simulation model, $\delta_{t}$ is used 
to calculate the cash flows that are paid out at the end of each time period as a function of the simulated project value.

As suggested in $\mathrm{BDH}$, we express the project value in terms of the project cash flows to allow greater flexibility in the modeling of the real options of the project. If we assume the risk free interest rate $r_{f}$ is constant, the present value at time $t$ is given by:

$$
V_{t}=X_{t}+\sum_{s=t+1}^{T}\left(1+r_{f}\right)^{-(s-t)} E\left(X_{s} \mid X_{t}\right)
$$

where $V_{t}$ is the stochastic value of the project at time period $t$, and $X_{t}=V_{t} \delta_{t}$ is the cash flow paid out at the end of period $t$, which reduces the project value in the subsequent time periods. Because of the payout of the cash flows in each period, the present value of the project will decrease throughout time if the cash flows are all positive. For a project with finite life, the final value of the project will be $X_{T}$, the last paid out cash flow at the terminal period $T$.

We now provide details regarding the four steps of the multi-factor real options valuation approach based on the G-IBT.

\section{Step 1. Calculate the Net Present Value}

This step is identical to the C\&A approach. Thus, with the fully risk neutral valuation approach, the expected present value of the project at time $0, E\left(V_{0}\right)$, is the discounted value of the expected project cash flows using a risk free rate without considering any managerial flexibility.

\section{Step 2. Estimate Parameters}

We apply the Monte Carlo simulation that describes the multiple uncertainties in the project cash flows to value the European-style options on the underlying project with different strike prices and maturities. This simulation may be based on the same spreadsheet used in Step 1 with 
a standard Excel add-in such as @ Risk or Crystal Ball. We use European call options to illustrate the technique, but European put options can be applied in a similar way.

Let there be $N$ simulated European call options $C_{t, i}$, each with a distinct strike price $K_{t, i}$, for each maturity time $t$. By risk neutral valuation, we have

$$
C_{t, i}=\left(1+r_{f}\right)^{-t} E\left[\max \left(V_{t}-K_{t, i}, 0\right)\right], \text { for } t=1, \ldots, T ; i=1, \ldots, N
$$

Evaluating the function $C_{t, i}$ with a large number of iterations and averaging the results produces

the Monte Carlo estimate $C_{t, i}^{\text {simulation }}$ for the European call option $C_{t, i}$. Since we are simulating the market information, we are not limited by the real market strike prices as in the financial application. Therefore, we can choose strike prices to provide more information about the tree structure. Since the underlying project values are usually log-spaced in a standard binomial tree, we suggest log-spaced strike prices that provide positive payoffs associated with their call options for each time period.

The implied distribution will converge to the actual underlying distribution when a sufficiently large number of options are available for use. Therefore, the more options simulated for the construction of implied binomial tree, the less sensitive the result will be to the choice of the strike prices.

\section{Step 3. Build the Binomial Tree}

The simulated European call option prices of all combinations of strike prices and maturities are used to calibrate the recombining implied binomial tree. Let $V_{t, j}$ denote the value of the project without options in period (column) $t$ and state (row) $j$, and $P_{t, j}$ denote the corresponding risk-neutral probabilities in the implied binomial tree, $t=0, \ldots, T, j=0, \ldots, t,(j=0$ is the lowest state). 
By risk-neutral valuation and the G-IBT approach, the European call option price $C_{t, i}{ }^{G-I B T}$ with strike price $K_{t, i}$ and maturity $t$, is given by

$$
C_{t, i}^{G-I B T}=\left(1+r_{f}\right)^{-t} \sum_{j=0}^{t}\left[P_{t, j} \max \left(V_{t, j}-K_{t, i}, 0\right)\right], \text { for } t=1, \ldots, T ; i=1, \ldots, N
$$

$C_{t, i}{ }^{G-I B T}$ should match with the corresponding simulated European call option price $C_{t, i}^{\text {simulation }}$.

Nonlinear programming methods are used to determine the risk neutral probabilities that minimize an objective function that optimizes the fit between the implied binomial tree and this simulated market information. These probabilities are then used to infer a unique, fully specified recombining binomial lattice consistent with these probabilities, and, hence, consistent with all the simulated European option prices.

The quadratic optimization (Rubinstein, 1994) minimizes the objective function of the sum of the squared deviations of posterior probabilities $P_{T, j}$ from a prior belief $P_{T, j}^{\prime}$ while satisfying the other constraints. The quadratic optimization problem for the G-IBT method is formulated as:

$$
\min _{P_{T, j}} \sum_{j}\left(P_{T, j}-P_{T, j}^{\prime}\right)^{2}
$$

subject to $0 \leq P_{T, j} \leq 1$ and $\sum_{j} P_{T, j}=1$, for $j=0, \ldots, T ; C_{t, i}{ }^{G-I B T}=C_{t, i}^{\text {simulation }}$, for $t=1, \ldots, T ; i=1, \ldots, N$; and the net present value $V_{0,0}$ from the implied binomial tree equal to $E\left(V_{0}\right)$. Additional constraints that relate the probabilities in period $t$ - 1 to those in period $t$, and that determine the transition probabilities of up and down moves in the binomial tree are also required, as explained in the Appendix.

Forcing option values calculated from the implied binomial tree to equal their values from the Monte Carlo simulation estimates may result in an infeasible solution. As discussed by 
Jackwerth and Rubinstein (1996), this problem can be overcome by allowing for a small error between these valuations and the use of an optimization routine that penalizes these deviations. The IBT approach is a recursive process starting from some ending period state prices $V_{T, j}$ of the project value. A description of the recursive process for the G-IBT approach is also provided in the Appendix.

Following Rubinstein (1994), we use the CRR lattice method to determine the $V_{T, j}$ since the CRR lattice naturally provides some log-spaced ending period state prices of the project value. In our example, we used log-spaced strike prices that equal to half of the ending period state prices from the GBM approximation. To build the CRR lattice, only the volatility of the project is needed and it can be estimated from a Monte Carlo simulation of the project returns $(\mathrm{C} \& \mathrm{~A}$, 2001; BDH, 2005 b).

Rubinstein (1994) suggested the use of an approximately lognormal distribution associated with the ending nodes of the CRR lattice as the prior belief for the ending nodal probability distribution. While the probability distribution of the ending period asset price is unknown in financial application, it can be estimated in the simulation-based real options application since we have simulated the ending period project value. Therefore, we can provide a more informative prior distribution rather than the lognormal prior. We assigned the simulated ending period project value to bins centered at the ending nodes of the CRR lattice and estimated the ending nodal probabilities. These simulated ending nodal probabilities $P_{T, j}^{\prime}$ are then used as the natural prior belief for the quadratic optimization that solves for ending nodal probabilities of the IBT tree. As the number of options becomes increasingly dense, the posterior distribution will become independent of the prior distribution (Rubinstein, 1994).

$\underline{\text { Step 4. Incorporate the Real Options }}$ 
This step is identical to the C\&A approach.

\section{Case Example}

We will illustrate the use of the IBT approach by calculating the real options value of a hypothetical mineral excavation example. In this example, we applied the G-IBT approach with the quadratic optimization method and compared the results with the GBM approximation (using a CRR lattice) with constant volatility and the BDH approximation with time varying volatilities. The example shows that the IBT approximation to the underlying stochastic process of project value is more accurate. The Longstaff and Schwartz Monte Carlo simulation approach is used as a benchmark.

We adapted the case example from the BDH paper for this discussion and adopted the fully risk neutral valuation approach. We assume that a mining production project has initial production level of 9 million tons. The initial variable operating cost is $\$ 10$ per ton and the initial mineral price is $\$ 25$ per ton. There is also a $\$ 5$ million per year fixed cost. The risk free interest rate is $5 \%$. In each year $t(t=1, \ldots, 10)$, the three relevant sources of uncertainty are the production level, the mineral price and the variable operating cost. We assume that the mining company determines the production level based on the market demand. The market demand and hence the production level $Y_{t}$ follows a triangular distribution Triang $(a, b, c)$, with minimum $a$, mode $b$, and maximum $c$, and $Y_{t}$ is serially correlated via the following process, $Y_{1} \sim \operatorname{Triang}(8,9,10)$, and $Y_{t} \sim \operatorname{Triang}\left(Y_{t-1}-1, Y_{t-1}, Y_{t-1}+1\right)$ for $t=2, \ldots 10$. The mineral price process follows a GBM process with mean drift (rate of change) of $0 \%$ and volatility of $15 \%$ and the variable operating cost process follows a GBM process with mean drift of $2 \%$ and volatility $10 \%$ respectively under the fully risk neutral approach. 
Cross sectional correlation could also be added without increasing the subsequent computational burden. For simplicity and for comparison, we consider the three stochastic factors to be mutually independent. We used @Risk for the Monte Carlo simulation.

We initially determined the expected value of the future cash flows as shown in Table 1. All values are in millions of dollars.

Table 1: Base Case (Expected Cash Flow for the Project)

\begin{tabular}{|c|c|c|c|c|c|c|c|c|c|c|c|}
\hline Year & 0 & 1 & 2 & 3 & 4 & 5 & 6 & 7 & 8 & 9 & 10 \\
\hline Production Level & & 9.00 & 9.00 & 9.00 & 9.00 & 9.00 & 9.00 & 9.00 & 9.00 & 9.00 & 9.00 \\
\hline Variable Op Cost Rate & & 10.2 & 10.4 & 10.6 & 10.8 & 11.0 & 11.3 & 11.5 & 11.7 & 12.0 & 12.2 \\
\hline Mineral Price & & 25.0 & 25.0 & 25.0 & 25.0 & 25.0 & 25.0 & 25.0 & 25.0 & 25.0 & 25.0 \\
\hline Revenues & & 225.0 & 225.0 & 225.0 & 225.0 & 225.0 & 225.0 & 225.0 & 225.0 & 225.0 & 225.0 \\
\hline Production Cost & & $(96.8)$ & $(98.6)$ & $(100.5)$ & (102.4) & $(104.4)$ & $(106.4)$ & (108.4) & $(110.4)$ & $(112.6)$ & $(114.7)$ \\
\hline Cash Flow & & 128.2 & 126.4 & 124.5 & 122.6 & 120.6 & 118.6 & 116.6 & 114.6 & 112.4 & 110.3 \\
\hline Profit Sharing & & $(32.1)$ & $(31.6)$ & $(31.1)$ & $(30.6)$ & $(30.2)$ & $(29.7)$ & $(29.2)$ & $(28.6)$ & $(28.1)$ & $(27.6)$ \\
\hline Net Cash Flows & & 96.2 & 94.8 & 93.4 & 91.9 & 90.5 & 89.0 & 87.5 & 85.9 & 84.3 & 82.7 \\
\hline PV of Cash Flows & 696.6 & 731.4 & 667.0 & 600.8 & 532.8 & 463.0 & 391.1 & 317.2 & 241.3 & 163.1 & 82.7 \\
\hline Cash Flow Payout Rate & & $13.1 \%$ & $14.2 \%$ & $15.5 \%$ & $17.3 \%$ & $19.5 \%$ & $22.8 \%$ & $27.6 \%$ & $35.6 \%$ & $51.7 \%$ & $100.0 \%$ \\
\hline
\end{tabular}

The present value of the expected cash flows is $\$ 696.6$ million, which is used as the best estimate of the current market value of the project without options. The required up-front investment is $\$ 180$ million, so the project's net present value is $\$ 516.6$ million. The cash flow payout rate $\delta_{t}$ for each period is calculated and is shown at the bottom of the table.

After running a large number $(10,000)$ of iterations, the Monte Carlo simulation provides the standard deviation of the project returns from period 0 to period 1 which is an estimate of the project volatility in period 1 . This estimate is $32.14 \%$ using the BDH (2005 b) approach. Since the time periods are one year in length in this example, this is the annualized volatility of the project returns in period 1 . This estimate is used as the constant volatility for generating the GBM approximation. 
We created the IBT and GBM approximations to the value of the underlying project using the steps outlined in the previous section. For simplicity, we built a ten period IBT and use 11 European call options for each period with the same log-spaced strike prices equal to half of the ending period state prices from the GBM approximation. Hence the tree was calibrated by a total of 110 European call options. The optimization was done with Excel solver. As explained in the Appendix, the optimization model also determines a weight function based on a parameter $\alpha$ that is used to calculate the implied nodal probabilities of the binomial tree in a recursive manner. The optimal weight function for the G-IBT was determined to be a piecewise linear concave weight function with the parameter $\alpha$ equal to 0.66 . With this concave weight function a path looping down first and then coming back up is more likely to be taken than a path looping up and then coming back down. This concave weight function implies that the project value has a more skewed distribution than the lognormal distribution assumption implied by the GBM approximation.

Figure 1 shows the histogram of the ending period cash flow probability distribution of the GBM approximation, the IBT tree, and of the simulated ending period project value. The risk neutral distribution of the IBT tree and the simulated project value are close based on a visual inspection, as we would expect since we used the simulated ending project value distribution as the prior distribution for the ending period distribution for the IBT approximation. In contrast with the simulated distribution of the ending period project value, the lognormal distribution under the GBM approximation is less skewed and has a much thinner left tail. Since the value of real options is usually driven by the tails of the distribution, a fat lower tail should lead to larger real option values for abandonment options, for example. 
Figure 1: Risk Neutral Distribution of Year 10

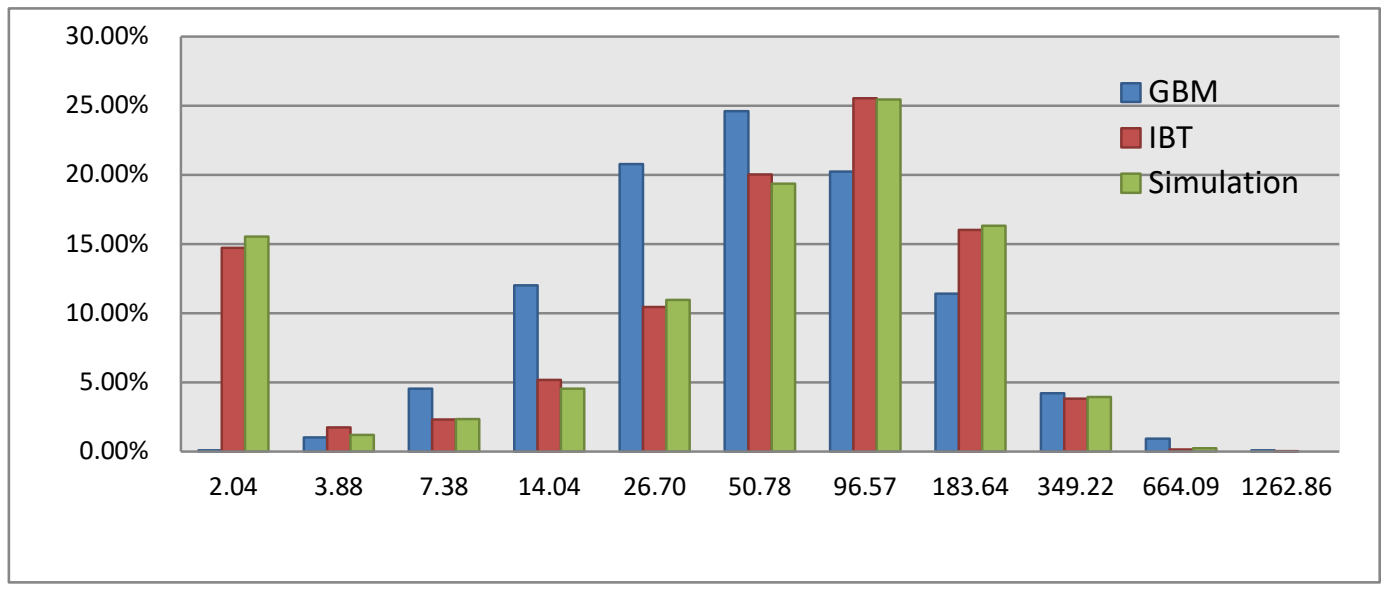

We can now calculate the value of any real option whose value is related to the value of the underlying multi-factor asset. Following the BDH example, we consider three alternatives at the end of year 5: continuing the project, buying out the partner's $25 \%$ share for $\$ 60$ million, or selling the decision maker's share for $\$ 100$ million (divesting). Given a binomial tree representation, this option can be evaluated by simply inserting a decision node in Year 5 that models the managerial flexibility that exists in the fifth year of the project. Other options or options in other periods could be incorporated in a similar manner.

The value of the project is determined by applying the usual procedures of dynamic programming implemented in a binomial lattice or tree and discounting the expected cash flows at the risk-free rate of return. If we incorporate the options directly into the IBT tree, the value of the project with options is estimated to be $\$ 766.27$. Therefore, the estimated value of the options to buy out or divest is worth $\$ 766.27-\$ 696.57=\$ 69.70$ million. In contrast, we obtain the project valuation with options of $\$ 751.77$ million by using the GBM approximation, which gives an estimate of this option value of $\$ 751.77-\$ 696.57=\$ 55.20$ million, a difference of $20.8 \%$ compared to the IBT approach. 
As illustrated in Smith (2005), we apply the Longstaff and Schwartz (2001) Monte Carlo simulation approach with linear regression. The project value with options calculated by simulation is $\$ 765.31$ million and the real options value is $\$ 765.31-\$ 696.57=\$ 68.74$ million. Because the Longstaff and Schwartz simulation approach determines the near-optimal exercise policy, it gives a close estimate to the real options value. We independently verified this benchmark by solving the problem using the Smith and Nau (1995) binomial tree approach and obtained the same answer to within one percent. The results from the IBT model, the GBM model and the Longstaff and Schwartz Monte Carlo simulation are shown in Table 2.

Table 2: Real Options Value by Different Approaches

\begin{tabular}{|l|l|l|l|}
\hline Project Valuation without Options & 696.57 & \multicolumn{2}{|l|}{} \\
\hline & $\begin{array}{l}\text { GBM } \\
\text { Model }\end{array}$ & $\begin{array}{l}\text { IBT } \\
\text { Model }\end{array}$ & $\begin{array}{l}\text { Longstaff } \\
\text { \& Schwartz }\end{array}$ \\
\hline Project Valuation with Options & 751.77 & 766.27 & 765.31 \\
\hline Value of Options & 55.20 & 69.70 & 68.74 \\
\hline Diff\% & $-19.70 \%$ & $1.39 \%$ & $0.00 \%$ \\
\hline
\end{tabular}

A close look at the probabilities of choosing each of the options provides more insight into the three approaches. Table 3 lists the probabilities of choosing each option from the GBM approximation, the IBT approximation, and the Longstaff and Schwartz simulation. Compared to the estimates from the Longstaff and Schwartz simulation, the GBM approximation presents significantly lower probabilities for the divest option, which is consistent with our previous observations regarding the skewness and thinner tail of the distribution of the project value determined by the GBM approximation. In contrast, the IBT tree provides closer estimates for the probabilities of the options that are selected.

Table 3: Probabilities of Choosing Options

\begin{tabular}{|l|l|l|l|}
\hline Option & $\begin{array}{l}\text { GBM } \\
\text { Approximation }\end{array}$ & $\begin{array}{l}\text { IBT } \\
\text { Approximation }\end{array}$ & $\begin{array}{l}\text { Longstaff } \\
\text { and Schwartz }\end{array}$ \\
\hline Buyout & $80.85 \%$ & $73.86 \%$ & $76.42 \%$ \\
\hline Continue & $15.92 \%$ & $8.88 \%$ & $10.36 \%$ \\
\hline Divest & $3.23 \%$ & $17.26 \%$ & $13.22 \%$ \\
\hline
\end{tabular}


The GBM approximation assumes the project value follows a GBM process over time with a constant volatility. We estimated the volatility of the project for each period (BDH, $2005 \mathrm{~b})$, and determined that the volatility varies over a small range from $32.14 \%$ to $35.87 \%$, so that it is approximately constant for this example.

To illustrate a case in which the volatility may change significantly over time, we changed the stochastic process for the mineral price from a GBM with constant volatility to one with stochastic volatility. Specifically, we assume the mineral price follows a Heston (1993) stochastic volatility process:

$$
\begin{aligned}
& d S_{t}=\theta S_{t} d t+\sqrt{v_{t}} S_{t} d W_{S} \\
& d v_{t}=\eta\left(\mu-\ln \left(v_{t}\right)\right) d t+\sigma v_{t} d W_{v}
\end{aligned}
$$

$S_{t}$ and $v_{t}$ denote the mineral price and the volatility of the mineral price at time $t$. The parameter $\theta$ is the mean for the mineral price process; $\eta$ determines the degree of reversion of the volatility to the mean $\mu$; $\sigma$ together with the level $v_{t}$ determines the volatility of the stochastic volatility. $d W_{S}$ and $d W_{v}$ are correlated Wiener process with constant correlation value $\rho$. In our example, we set the parameters of the risk neutral mineral price process as follows, $\theta=0$, $\eta=0.5, \mu=\ln \left(0.2^{2}\right), \sigma=0.2, \rho=0$

With the stochastic volatility, the project value is more volatile. The volatility changes from $37.24 \%$ to $62.36 \%$ from year 1 to year 10 . Once again, we created the IBT and GBM approximations to the value of the underlying project and evaluated the same real options in the previous example. For the GBM approximation, we assumed that the volatility stayed constant over time at its estimated value of $37.24 \%$ for year 1 . As an extension of the GBM approximation, BDH (2005 b) proposed a non-recombining binomial tree approximation with 
time varying volatilities assuming a GBM process for each time period. The time varying volatilities can be estimated with Monte Carlo simulation as discussed in BDH (2005 b). We built the BDH tree with time varying volatilities as another comparison to the IBT approximation. Table 4 lists the real options values and the probabilities of choosing each of the options using different models. Once again, the Longstaff and Schwartz results are used as the benchmark.

Table 4: Real Options Value by Different Approaches and Probabilities of Choosing Options

\begin{tabular}{|l|l|l|l|l|}
\hline Project Valuation without Options & 696.57 & \multicolumn{4}{|l|}{} \\
\hline & $\begin{array}{l}\text { GBM } \\
\text { Approximation }\end{array}$ & $\begin{array}{l}\text { BDH } \\
\text { Approximation }\end{array}$ & $\begin{array}{l}\text { IBT } \\
\text { Approximation }\end{array}$ & $\begin{array}{l}\text { Longstaff } \\
\text { and Schwartz }\end{array}$ \\
\hline Project Valuation with Options & 754.69 & 759.5 & 775.42 & 774.45 \\
\hline Value of Options & 58.12 & 62.94 & 78.85 & 77.89 \\
\hline Diff\% & $-25.37 \%$ & $-19.19 \%$ & $1.24 \%$ & $0.00 \%$ \\
\hline Probabilities of Choosing Options & \multicolumn{5}{|l}{} \\
\hline Buyout & $77.74 \%$ & $67.51 \%$ & $66.99 \%$ & $68.66 \%$ \\
\hline Continue & $0.00 \%$ & $7.32 \%$ & $9.28 \%$ & $11.44 \%$ \\
\hline Divest & $22.26 \%$ & $25.17 \%$ & $23.73 \%$ & $19.90 \%$ \\
\hline
\end{tabular}

The result from the GBM approximation underestimates the project value by $25.37 \%$ and results in a set of significantly different probabilities of choosing each option compared to the results from the Longstaff and Schwartz simulation. We can see that the GBM assumption does not provide good approximations for the stochastic processes associated with the project values in the two versions of this example, and therefore the estimates of the real options values based on this approximation are not accurate. The BDH model with time varying volatilities performs better than the GBM approximation with a $19.19 \%$ difference from the simulation result for the real options value and some improvement for the probabilities of choosing each option. The IBT model gives the closest estimate of the value of the options, within $1.24 \%$ of the simulation result, and closer estimates of two of the probabilities of the options selected. The IBT approach provides more modeling flexibility to the project value distributions and therefore the IBT approach provides an accurate estimate of the real options value. 


\section{Conclusion}

We have illustrated the use of the IBT method to solve multi-factor real options valuation problems by combining Monte Carlo simulation and the use of a binomial lattice. This approach implements multi-factor real options valuation techniques using risk neutral probabilities inferred from the simulated market information. By constructing the recombining implied binomial tree that is consistent with the simulated European options prices, this algorithm relaxes the distribution assumption for the underlying project value, and provides a computationally efficient and accurate way to value many high dimensional real options problems with generalized diffusion processes.

The IBT approximation does share some of the limitations of the C\&A approach. The aggregation of multiple project uncertainties (e.g., price, quantity, and cost) into the single measure of project performance, the cash flows, does introduce some restrictions on the use of this approach. This approach is well suited to model project options with the effect of changing the "scale" of the cash flows, such as the option to increase or to reduce the ownership share of a project by some percentage, or to abandon it. In other cases, it may be possible to adapt this approach to situations to handle more complex situations by approximating the stochastic process for cash flows given different scenarios.

For example, the valuation of an oil well may include the uncertainty of the initial production rate, modeled as a discreet distribution. Changes in the initial production rate will have an effect on the stochastic process for the cash flows that might be expected from the project, and so an approximation of the stochastic cash flow with the IBT may be needed for each production rate scenario. A similar strategy could be used to approximate different stochastic processes if, for example, the option under consideration involves a project delay 
where new information may be obtained that will have an impact on one or more of the project uncertainties.

We do subscribe to the point of view that there is no one "magic bullet" for solving real options problems, and believe that some real options problem may be more suited to an analysis using alternative methods. For example, we used the Longstaff and Schwartz simulation model as a benchmark for evaluating our alternative binomial tree approximation methods, and recognize that it might be used instead to value simple real options such as the ones incorporated in these examples. However, its complexity grows with the number of decisions and alternatives and the need to run more regressions for additional decision points, working backward from the last set of decisions, so there may be challenges associated with its use as well for more complex real options problems.

See Smith (2005) for a discussion of some alternative real options methods, and Borison (2005), Smith (2005) and Brandao, et al (2005a, 2005b) for a discussion of their pros and cons. The ultimate choice of a method of analysis for this class of problems will depend to a large degree on the characteristics of the problem itself. These characteristics may include the number of uncertainties and their relationships, the number of options and their complexity, the number of time periods that need to be modeled, and so forth. In addition, there may also be a matter personal taste related to computational tools and to such issues as the preference for a visual model representation or the commonly accepted analytical tools used within an organization. 


\section{References}

1. Aronld, Tom, Timothy F. Crack, and Adam Schwartz. "Valuing Real Options Using Implied Binomial Trees and Commodity Futures Options.” The Journal of Futures Markets 27, (2007): 203-226

2. Borison, Adam, "Real Options Analysis: Where are the Emperor's Clothes ?", Journal of Applied Corporate Finance, 17.2 (2005), 17-31.

3. Brandao, Luiz E., James S. Dyer, and Warren J. Hahn. "Using Binomial Decision Trees to Solve RealOption Valuation Problems." Decision Analysis 2.2 (2005a): 69-88.

4. Brandao, Luiz E., James S. Dyer, and Warren J. Hahn. "Response to Comments on Brandao Et Al." Decision Analysis 2.2 (2005b): 103-09.

5. Copeland, Thomas E., and Vladimir Antikarov. "Real Options: A Practitioner's Guide." Texere, 2001.

6. Derman, Emanuel, and Iraj Kani. "Riding on a Smile." Risk 7.2 (1994): 32-39.

7. Dupire, Bruno."Pricing with a Smile." Risk 7.1 (1994): 18-20.

8. Godinho, Pedro. "Monte Carlo Estimation of Project Volatility for Real Options Analysis." Journal of Applied Finance 16.1 (2006): 15-30.

9. Hahn, Warren J., and James S. Dyer. "Discrete Time Modeling of Mean-Reverting Stochastic Processes for Real Option Valuation." European Journal of Operational Research 184.2 (2008): 534-48.

10. Heston, Steven. "A Closed-Form Solution for Options with Stochastic Volatility with Applications to Bond and Currency Options". The Review of Financial Studies 6.2 (1993): 327-343

11. Jackwerth, Jens C. "Generalized Binomial Trees”. Journal of Derivatives 5, (1997): 7-17.

12. Jackwerth, Jens C. "Implied Binomial Trees: A Literature Review". Journal of Derivatives 7 (1999): 66-82.

13. Jackwerth, Jens C., and Mark Rubinstein. "Recovering Probability Distributions from Option Prices." The Journal of Finance 51.5 (1996): 1611-31.

14. Lim, Kian Guan, Da Zhi. "Pricing Options Using Implied Trees: Evidence from FTSE-100 Options." The Journal of Futures Markets 22.7 (2002): 601-626.

15. Longstaff, Francis A., and Eduardo S. Schwartz."Valuing American Options by Simulation: A Simple Least-Squares Approach." The Review of Financial Studies 14 (2001): 113-47.

16. Rubinstein, Mark. "Implied Binomial Trees." The Journal of Finance 49.3 (1994): 771-818.

17. Smith, James E. "Alternative Approaches for Solving Real-Options Problems: (Comment on Brandao Et Al. 2005)." Decision Analysis_2.2 (2005): 89-102.

18. Smith, James E., and Kevin F. McCardle. "Options in the Real World: Lessons Learned in Evaluating Oil and Gas Investments," Operations Research, 47 (1999), 1-15.

19. Smith, James E., and Robert F. Nau. "Valuing Risky Projects: Option Pricing Theory and Decision Analysis." Management Science 41.5 (1995): 795-816. 


\section{Appendix}

The IBT approach approximates the ending period project value as $T+1$ discrete values $V_{T, 0}, V_{T, 1}, \ldots, V_{T, T-1}, V_{T, T}$. The risk-neutral ending nodal probabilities corresponding to these project values are $P_{T, 0}, P_{T, 1}, \ldots, P_{T, T-1}, P_{T, T}$.

The nodal probabilities $P_{t, j}$ and state prices $V_{t, j}$ for the previous periods are determined recursively. The G-IBT approach uses a weight function to assign a particular portion of the nodal probability going to the lower preceding node. In this paper, we follow Jackwerth (1997) and use the piecewise linear generalized weight function given below to illustrate the technique and refer the readers to Lim and Zhi (2002) for further discussion on other proposed linear and nonlinear weight functions.

$$
W(x)=\left\{\begin{array}{ccc}
2 \alpha x & \text { for } & x \in[0,0.5] \\
2(1-\alpha)(x-0.5)+\alpha & \text { for } & x \in[0.5,1]
\end{array}\right.
$$

$\alpha \in[0,1]$ is a variable to be determined by the optimization method to best match the tree structure with the simulated European option prices. The path independence assumption of the R-IBT approach is the special case of the linear weight function with $\alpha=0.5$.

Together with the ending nodal probabilities and state prices, the entire binomial tree can be effectively summarized through the weight function,

$$
P_{t-1, j}=W\left(\frac{j+1}{t}\right) P_{t, j+1}+\left(1-W\left(\frac{j}{t}\right)\right) P_{t, j}
$$

The transition probabilities of an up move over the next time step at the preceding nodes are

$$
q_{t-1, j}=\frac{W\left(\frac{j+1}{t}\right) P_{t, j+1}}{P_{t-1, j}}
$$


The project value at the preceding node $V_{t-1, j}$ is then calculated from the risk-neutral valuation formula, with $V_{t, j}$ and $\delta_{t}$ defined earlier in the paper.

$$
V_{t-1, j}=\frac{1}{\left(1-\delta_{t-1}\right)}\left(q_{t-1, j} V_{t, j+1}+\left(1-q_{t-1, j}\right) V_{t, j}\right)
$$

Following the same logic, we recursively move back to the first period and calculate the corresponding nodal probabilities and state prices of the whole tree. 